www. revistad yo. com

\title{
Robust Car Sequencing Problem: Description, models and metrics
}

\author{
Joaquín Bautista-Valhondo \\ Recibido: 8 de Marzo de 2019 / Aceptado: 10 de Abril de 2019
}

\section{Summary}

The concept of partial demand uncertainty is introduced from the car sequencing problem (CSP) and incorporates special vehicle fleets in a demand plan. After establishing the working hypotheses with fleets, a mixed integer linear programming model, robust car sequencing problem (r-CSP), is proposed to satisfy the maximum number of CSP restrictions. Subsequently, multi-sequence production and metrics to evaluate its robustness are defined. The r-CSP considers various demand scenarios and functions to measure the excess of optional requirements in production programs. These functions are valid as objectives in optimization problems and as multi-sequence pro-duction robustness metrics.

\section{Keywords}

Assembly lines, Sequencing, Car Sequencing Problem, Vehicle Fleets, Robust Optimization, Production Mix, Demand uncertainty.

\section{Introduction}

In the manufacturing environment of the automotive sector, product-oriented manufacturing systems are typical. In these environments, the manufacture of a product (engine, stamping part, framework, welded bodywork, painted bodywork, chassis, dress, etc.) is conceived as a set of consecutive stages or processes that add value by converting raw materials into finished products (vehicle). These manufacturing processes can be highly automated using robots or require a large number of human resources owing to the complexity of some operations.

Product-oriented manufacturing leads to flexible production systems composed of cells and the so-called production and assembly lines that are composed of modules or work stations.

Among the different types of production lines [Battaïa and Dolgui (2013)], mixed-model assembly lines are found that can manufacture variants of a product (SUVs, 4 x 4, vans, etc.) with diverse options (long or short framework, sunroof or rigid roof, tinted or blind-metal window, etc.), without requiring substantial changes in staff or instruments in the work stations.

In the mixed-model production lines, two categories of problems can be distinguished:
P1. Production line balancing: Problems oriented to the efficient allocation of a set of product assembly tasks to a set of workstations arranged in series, respecting a number of temporal (cycle time), spatial (available area) [Chica et al. (2016)] and contingency (ergonomic risk) constraints [Bautista et al. (2016)].

P2 Sequencing of mixed products: Problems oriented to establishing the manufacturing order (entry to the line) of the product units according to one or more criteria, one or more demand plans, and a timeframe to execute them [Boysen et al. (2009)].

Owing to the complexity of both categories of problems, the standard practice, both in industry and academia, is to solve them consecutively: first, line balancing is established; subsequently, the most appropriate product sequence is determined in accordance with the imposed conditions.

The objectives, not necessarily excluded, that are considered when sequencing the models, respond to several productive concerns [Bautista and Cano (2011)]. Among the objectives are the following:

o1 Maximize the number of units completed in the production line. Hence, efforts are conducted to reduce the inert time of the workers, the unnecessary waiting, and the production losses caused by workload excess (overload) in the stations [Yano and Rachamadugu (1991), Cano-Belmán et al. (2010), Bautista et al. (2012)].

o2 Maximize the satisfaction of productive restrictions. These restrictions are related to the critical components of the vehicles or to the standard options included in the model catalogue [Parrello et al. (1986), Bautista et al. (2008.a)]. 
03 Minimize the stock levels of the components, both in the production plant and in the supply chain. Given the regularity in the production [Bautista and Alfaro (2017)], it is intended to maximize the consistency of the production rates of products [Bautista et al. (1997), Corominas and Moreno (2003)] and the rates of component consumption [Monden (1994), Bautista et al. (1996)].

Among the sequencing problems corresponding to objective (o2), the car sequencing problem (CSP) can be found, whose original purpose [Parrello et al. (1986)] consists in to establish a sequence $\pi(\mathrm{T})$ of different type T vehicles in function of their optional elements. The nature of the problem is highly combinatorial [Gent (1998), Kis (2004)], for which its resolution has traditionally focused on the use of metaheuristics [Gottlieb et al. (2003), Bautista et al. (2008.b), Ribeiro et al. (2008), Morin et al. (2009), Siala et al. (2015)].

In the CSP, the vehicles are classified in a set $\mathrm{I}$, of which $\mathrm{d}_{\mathrm{i}}$ are of the type $\mathrm{i}(\forall \mathrm{i} \in \mathrm{I})$. Obviously, the sequence $\pi(\mathrm{T})$ must be constructed in harmony with the vehicle demand plan that is symbolized using the vector $d^{r}=\left(\mathrm{d}_{1}, \ldots, \mathrm{d}_{|I|}\right)$, satisfying $\mathrm{D} \equiv \mathrm{T}=\sum_{\forall \mathrm{i}} \mathrm{d}_{\mathrm{i}}$.

The typology of these vehicles is a function of the presence of optional elements - classified in a set J-. Therefore, a vehicle type $\mathrm{i}(\mathrm{i}=1, . ., \mid \mathrm{I})$ ) may or may not contain the option $\mathrm{j}(j=1, . .,|J|)$; this is reflected by the parameters $n_{j, \mathrm{i}}$ that adopt the value 1 if the option $j \in J$ is present in the vehicle type $i \in I$, and the value 0 otherwise.

These optional elements are the focus of the problem, because their requirements by a group of vehicles added consecutively to the production line is limited. These limitations can be represented through the ratios $\mathrm{p} / \mathrm{q}_{\mathrm{j}}(\forall \mathrm{j} \in \mathrm{J})$ that symbolize the following: given the option $j \in J$, its requirement by the vehicles contained in any segment of the sequence $\pi(T)$ with a length $q_{j}$ (i.e., every segment with $q_{j}$ consecutive production cycles) must be less than or equal to the value $\mathrm{p}_{\mathrm{j}}$; colloquially, as a maximum, the option $j \in J$ will be present $p_{j}$ times in each cycle $\mathrm{q}_{\mathrm{j}}$.

Under these conditions, the original CSP consists of obtaining a sequence $\pi(T)$ that satisfies all the restrictions on the optional element requirements. If the above is impossible, the objective of the problem is to ensure that the sequence $\pi(T)$ satisfies the greatest number of such restrictions.

The remainder of this paper is structured as follows. In section 2, the new problem incorporating fleets of special vehicles and robustness to the original CSP are presented, where some peculiarities of these fleets are described. In section 3, the working hypotheses for the problem are stated, a nomen- clature is presented, a basic optimization model based on the mixed integer linear programming (MILP) is proposed, and the multi-sequence concept resulting from the exploitation of the proposed model is introduced. In section 4, several metrics to evaluate the non-robustness and robustness of a multi-sequence of vehicles is offered. In section 5, the elementary variants on the r-CSP basic model (robust-CSP) are gathered: mono-objective functions based on the weighting of the excess of optional elements requirements and in the robustness metrics of a of production multi-sequence are proposed, as well as functions to address the multi-objective optimization in the problem. Finally, section 6 presents a synthesis and some considerations regarding this work.

\section{CSP with fleets of special vehicles}

Large automotive companies contain manufacturing and assembly lines that can accommodate their production programs, e.g., the insertion of non-regular or out-of-catalogue vehicles.

These special vehicles, termed fleets herein, are distinguished from the typical or regular vehicles for various reasons, and present some peculiarities:

a. They are vehicles with demand under contract and are not part of the sales forecasts prepared by the commercial department.

b. Their destination varies and typically corresponds to public service organizations: ambulances, vehicles destined to defense, armed forces vehicles, fire trucks, police patrol cars, forest guard vehicles, etc.

c. These fleets require non-typical components that, when incorporated to the vehicles in the production line, generate additional operations that require greater processing times than standard operations.

d. The non-typical components of special vehicles also depend on the type of fleet (ambulance, police patrol cars, forest guard vehicles, etc.). Therefore, the incorporation of a special vehicle type to the daily production program will cause differences in the consumption of components, for the requirement of tools and equipment, in the workloads and in the line supplies.

e. Logically, the inclusion of special vehicles in the existing production programs will be limited by a number or by a proportion of those over the total. Typically, this proportion oscillates between $10 \%$ and $20 \%$ of the daily production of vehicles. 
f. To facilitate the production management, the daily global manufacture of special vehicles is set at a constant value; however, the partial production of each fleet type may vary by day. Meanwhile, the production of typical vehicles is not subject to daily demand alterations; in fact, their daily demand is stable and regular, and is aimed at satisfying the weekly or monthly demand plan.

g. The possible variation in the daily demand of the special vehicles fleets generates uncertainty when preparing the production schedule; consequently, the manufacturing sequence of vehicles will be subjected to uncertainty. Obviously, it is impossible to establish a manufacturing sequence if the elements that constitute it are unknown.

h. An alternative to handle the demand variation in the special vehicles fleets is to assume different demand scenarios. These scenarios must be realistic and in accordance with the production capacity and history of the manufacturing plant.

Formally, the demand scenarios will be grouped into a set $\mathrm{E}$ of elements $\varepsilon \in \mathrm{E}$. To define the scenario $\varepsilon \in \mathrm{E}$, the demand vector $\overrightarrow{d_{\varepsilon}}=\left(\mathrm{d}_{1 \varepsilon}, \ldots, \mathrm{d}_{|| \mid \varepsilon}\right)$, and the mixed-production vector $\overrightarrow{\lambda_{\varepsilon}}=\left(\lambda_{1 \varepsilon}, \ldots, \lambda_{|| \mid \varepsilon}\right)$ will be used, where $\mathrm{d}_{\mathrm{i} \varepsilon}$ and $\lambda_{\mathrm{i} \varepsilon}$ are the number of vehicles $i \in I$ and their proportions in the plan $\varepsilon \in \mathrm{E}$, respec-tively. For coherence reasons, $\overrightarrow{\lambda_{\varepsilon}}=\overrightarrow{d_{\varepsilon}} / \mathrm{D}_{\varepsilon} \mathrm{y}$ $\mathrm{D}_{\varepsilon}=\sum_{\mathrm{i} \in \mathrm{I}} \mathrm{d}_{\mathrm{i} \varepsilon}$, must be satisfied.

It is noteworthy that the ordinary CSP consists in obtaining a sequence $\pi(\mathrm{T})$ that satisfies the largest number of restrictions on the optional elements requirements, while the CSP with fleets of special vehicles, which will be termed r-CSP herein, consists of obtaining a set of sequences $\pi_{\varepsilon}(T)$, one for each plan $\varepsilon \in E$, that satisfy the largest number of such restrictions; in addition, these sequences resemble each other closely [Bautista (2016)].

\section{Basic CSP model with fleets of special vehicles}

This section describes the proposal of a basic model for the CSP with fleets of special vehicles: a problem that will also be referred to as a robust version of the CSP (r-CSP).
First, the working hypotheses about the new problem is stated; next, a nomenclature to define and describe the variables and parameters that intervene in the model is proposed; subsequently, the r-CSP model is formulated within the framework of MILP; finally, the concept of multi-sequence production is defined as a solution representation form of any instance of the problem, for a set of demand plans that must be satisfied simultaneously as much as possible.

\section{HYPOTHESIS:}

1. Two families of vehicles are available: (i) the family of typical or regular vehicles, represented by the set of types $I_{X}$; (ii) the family of special vehicles fleets, represented by the set of types $\mathrm{I}_{\mathrm{X}}$.

2. The total number of regular vehicles $\mathrm{D}_{\mathrm{x}}$, corresponding to a working day, is identical for all the demand plans $\varepsilon \in \mathrm{E}$.

3. The total number of special fleet vehicles $\mathrm{D}_{\mathrm{X}}$, corresponding to a working day, is identical for all the demand plans $\varepsilon \in E$.

4. Consequently, the total number of vehicles $\mathrm{T}\left(\mathrm{T} \equiv \mathrm{D}=\mathrm{D}_{\mathrm{X}}\right.$ $+\mathrm{D}_{\mathrm{X}}$ ) that corresponds to a working day, is identical for all the demand plans $\varepsilon \in E$.

5. The demand for a regular vehicle $i \in I_{x}$, corresponding to a working day, is identical for all the demand plans $\varepsilon \in \mathrm{E}$. That is, if $\mathrm{i} \in \mathrm{I}_{\mathrm{X}}$, then $\mathrm{d}_{\mathrm{i}, \varepsilon}=\mathrm{d}_{\mathrm{i}} \forall \varepsilon \in \mathrm{E}$ is satisfied.

6. The demand for a special fleet vehicle $i \in I_{X^{\prime}}$, corresponding to a working day, may change for two different demand plans $\left\{\varepsilon, \varepsilon^{\prime}\right\} \subseteq$ E.

7. To minimize the number of changes in the production line (e.g.,robots, instruments, tools, shelves, personnel, etc.), the manufacturing sequences $\pi_{\varepsilon}(\mathrm{T})$ and $\pi_{\varepsilon^{\prime}}(\mathrm{T})$ must be as similar as possible for every pair of plans $\left\{\varepsilon, \varepsilon^{\prime}\right\} \subseteq$ E. Symbolically, $\pi_{\varepsilon}(\mathrm{T}) \approx \pi_{\varepsilon^{\prime}}(\mathrm{T}) \quad \forall\left\{\varepsilon, \varepsilon^{\prime}\right\} \subseteq \mathrm{E}$.

8 As an immediate consequence of the previous hypothesis, all regular vehicles will be forced to occupy individually and by type; the same positions apply in all the sequences $\pi_{\varepsilon}(T)(\forall \varepsilon \in E)$. 
Joaquín Bautista-Valhondo / Dirección y Organización 68 (2019) 105-116

Table 1 Nomenclature - parameters

Table 2 Nomenclature - variables

\begin{tabular}{|c|c|}
\hline Nomenclature & Parameters \\
\hline$I_{x}$ & Set of regular or standard types of vehicles $\left(\mathrm{i}=1, . .,\left|\mathrm{I}_{X}\right|\right)$. \\
\hline$I_{X^{\prime}}$ & Set of special types of vehicles (fleets) $\left(\mathrm{i}=\left|\mathrm{I}_{X}\right|+1, \ldots,\left|\mathrm{I}_{X}\right|+\left|\mathrm{I}_{X}\right|\right)$. \\
\hline I & Set of vehicle types: $I=I_{X} \cup I_{X^{\prime}}(i=1, . .,|I|)$. \\
\hline $\mathrm{J}$ & Set of component parts or optional elements $(\mathrm{J}: \mathrm{j}=1, \ldots, \mathrm{J} \mid)$. \\
\hline E & Set of scenarios or demand plans $(E: \varepsilon=1, \ldots,|E|)$. \\
\hline $\overrightarrow{d_{\varepsilon}}, \mathrm{D}$ & $\begin{array}{l}\text { Demand plan vector } \varepsilon \in \mathrm{E}: \overrightarrow{d_{\varepsilon}}=\left(\mathrm{d}_{1, \varepsilon} \cdots, \mathrm{d}_{||, \varepsilon}\right) \text { and total demand of vehicles in a workday: } \mathrm{D} \equiv \mathrm{T}=\sum_{\mathrm{yi}} \mathrm{d}_{\mathrm{i}, \varepsilon} \text {, } \\
\text { identical in all plans } \varepsilon \in \mathrm{E} \text {. }\end{array}$ \\
\hline $\overrightarrow{\lambda_{\varepsilon}}$ & Mixed-production plan vector $\varepsilon \in \mathrm{E}: \overrightarrow{\lambda_{\varepsilon}}=\left(\lambda_{1, \varepsilon} \cdots, \lambda_{\|, \varepsilon}\right): \overrightarrow{\lambda_{\varepsilon}}=\overrightarrow{d_{\varepsilon}} / \mathrm{D}$ \\
\hline $\mathrm{n}_{\mathrm{j}, \mathrm{i}}$ & $\begin{array}{l}\text { Binary parameter that adopts the value } 1 \text { if the optional element } j \in \mathrm{J} \text { is present in the vehicle type } \mathrm{i} \in \mathrm{I} \text {, and the } \\
\text { value } 0 \text { otherwise. }\end{array}$ \\
\hline $\mathrm{p}_{\mathrm{j}} / \mathrm{q}_{\mathrm{j}}$ & $\begin{array}{l}\text { CSP ratios that symbolize the requirement of the optionj } \in J \text { by the vehicles contained in any segment of the } \\
\text { sequences } \pi_{\varepsilon}(T)(\forall \varepsilon \in E) \text { with a length } \mathrm{q}_{j} \text {; must be less than or equal to the value } \mathrm{p}_{\mathrm{j}} \text {. }\end{array}$ \\
\hline $\mathrm{c}_{\mathrm{j}, \mathrm{t}, \mathrm{\varepsilon}}$ & $\begin{array}{l}\text { Cost or weight attributed to the segment of consecutive cycles }\left[t-q_{j}+1, t\right] \text { of the sequence } \pi_{\varepsilon}(T) \text {, linked to } \\
\text { the demand plan } \varepsilon \in E \text {, when the requirement of the option } j \in J \text { is greater than } p_{j} \text { in that segment. In this basic } \\
\text { model, all the unit costs will be assumed: } c_{i, t, \varepsilon}=1\left(\forall j \in J, \forall t \in\left[q_{j} T\right], \forall \varepsilon \in E\right) \text {. }\end{array}$ \\
\hline Nomenclature & Variables \\
\hline$\pi_{\varepsilon}(\mathrm{T})$ & $\begin{array}{l}\text { Complete sequence of vehicles } \pi_{\varepsilon}(\mathrm{T})=\left(\pi_{1, \varepsilon}, \ldots, \pi_{\mathrm{T}, \mathrm{T}}\right) \text { of the plan } \varepsilon \in \mathrm{E} \text {. The partial sequences of } \pi_{\varepsilon}(\mathrm{T}) \text { will be } \\
\text { represented as } \pi_{\varepsilon}(\mathrm{t})=\left(\pi_{1, \varepsilon}, \ldots, \pi_{\mathrm{t}, \varepsilon}\right) \subseteq \pi_{\varepsilon}(\mathrm{T}), \forall \mathrm{t} \in[1, \mathrm{~T}] \text {. The symbols } \pi_{\varepsilon}(\mathrm{t}) \text { y } \pi_{\varepsilon}(\mathrm{T}) \text { are also used as parameters. }\end{array}$ \\
\hline $\mathrm{x}_{\mathrm{i}, \mathrm{t}}$ & $\begin{array}{l}\text { Binary variable that adopts the value } 1 \text { if a regular vehicle unit } \mathrm{i} \in \mathrm{I}_{\mathrm{X}} \text { is assigned to the position } \mathrm{t}(\mathrm{t}=1, \ldots, \mathrm{T}) \text { of } \\
\text { the sequences } \pi_{\varepsilon}(\mathrm{T}) \text { of the plan } \varepsilon \in \mathrm{E} \text {, and adopt the value } 0 \text { otherwise. }\end{array}$ \\
\hline $\mathrm{x}_{\mathrm{i}, \mathrm{t} \mathrm{e}^{\prime}}$ & $\begin{array}{l}\text { Binary variable that adopts the value } 1 \text { if a special vehicle unit } \mathrm{i} \in \mathrm{I}_{\mathrm{X}^{\prime}} \text { is assigned to the position } \mathrm{t}(\mathrm{t}=1, \ldots, \mathrm{T}) \text { of } \\
\text { the sequence } \pi_{\varepsilon}(\mathrm{T}) \text { of the plan } \varepsilon \in \mathrm{E} \text {, and it is } 0 \text { otherwise. }\end{array}$ \\
\hline $\mathrm{X}_{\mathrm{i}, \mathrm{t}}$ & $\begin{array}{l}\text { Number of regular vehicle unit type } i \in I_{X} \text { contained in all partial sequences } \pi_{\varepsilon}(t) \subseteq \pi_{\varepsilon}(T) \text { of all plans } \varepsilon \in E \text {. Its } \\
\text { calculation is performed as follows: } X_{i, t}=\sum_{t=1}^{t} X_{i, t}, \forall i \in I_{X}, \forall t \in[1, T]\end{array}$ \\
\hline $\mathrm{X}_{\mathrm{i}, \mathrm{t}, \varepsilon^{\prime}}$ & $\begin{array}{l}\text { Number of special vehicle unit type } \mathrm{i} \in \mathrm{I}_{X^{\prime}} \text { contained in the partial sequence } \pi_{\varepsilon}(t) \subseteq \pi_{\varepsilon}(T) \text { of the plan } \varepsilon \in \mathrm{E} \text {. } \\
\text { Its calculation is performed as follows: } \mathrm{X}_{\mathrm{i}, \mathrm{t}, \mathrm{s}}^{\prime}=\sum_{\mathrm{t}=1}^{\mathrm{t}} \mathrm{x}_{\mathrm{i}, \mathrm{i}, \varepsilon}^{\prime} \forall \mathrm{i} \in \mathrm{I}_{\mathrm{X},}, \forall \mathrm{t} \in[1, T], \forall \varepsilon \in \mathrm{E}\end{array}$ \\
\hline $\mathrm{Y}_{\mathrm{j}, \mathrm{s}, \mathrm{s}}$ & $\begin{array}{l}\text { Number of times that the option } j \in J \text { is required by the regular and special vehicles contained in the partial } \\
\text { sequence } \pi_{\varepsilon}(t) \subseteq \pi_{\varepsilon}(T) \text { of the plan } \varepsilon \in E \text {. It is calculated as follows: } Y_{j, t, \varepsilon}=\sum_{i, \in|\times|} n_{j, i} X_{i, t}+\sum_{i \in \mid x} n_{j, i} X_{i, t, \varepsilon}^{\prime} \forall j \in- \\
J, \forall t \in[1, T], \forall \varepsilon \in E \text {. For convenience, it will be calculated as follows: } Y_{i, 0, \varepsilon}=0 \quad \forall j \in J, \forall \varepsilon \in E\end{array}$ \\
\hline $\mathrm{z}_{\mathrm{j}, \mathrm{t}, \mathrm{s}}$ & $\begin{array}{l}\text { Binary variable that adopts the value } 1 \text { if the requirement of option } j \text { is greater than the value } \mathrm{p}_{\mathrm{j}} \text { in the segment } \\
{\left[\mathrm{t}-\mathrm{q}_{\mathrm{j}}+1, \mathrm{t}\right] \text { of the sequence } \pi_{\varepsilon}(\mathrm{T}) \text {, and it is } 0 \text { otherwise }\left(\forall j \in J, \forall t \in\left[\mathrm{q}_{\mathrm{j}} T\right], \forall \varepsilon \in \mathrm{E}\right) \text {. For convenience, the symbols }} \\
\mathrm{z}_{\mathrm{j}, \mathrm{s}, \mathrm{s}} \text { will also be used as parameters when the sequence } \pi_{\varepsilon}(\mathrm{T}) \text { is known. }\end{array}$ \\
\hline
\end{tabular}


FORMULATION - BASIC r-CSP MODEL:

$$
\min Z=\sum_{j \in J} \sum_{t \in\left[q_{j}, T\right]} \sum_{\varepsilon \in \mathrm{E}} z_{j, t, \varepsilon} \Leftrightarrow \max Z^{\prime}=\sum_{j \in J} \sum_{t \in\left[q_{j}, T\right]} \sum_{\varepsilon \in \mathrm{E}}\left(1-z_{j, t, \varepsilon}\right)
$$

Subject to:

$$
\begin{aligned}
& \sum_{i \in I_{X}} x_{i, t}+\sum_{i \in I_{X^{\prime}}} x_{i, t, \varepsilon}^{\prime}=1 \forall t \in[1, T], \forall \varepsilon \in \mathrm{E} \\
& \sum_{t \in[1, T]} x_{i, t}=d_{i} \forall i \in I_{X} \\
& \sum_{t \in[1, T]} x_{i, t, \varepsilon}^{\prime}=d_{i, \varepsilon} \quad \forall i \in I_{X^{\prime}}, \forall \varepsilon \in \mathrm{E} \\
& X_{i, t}-\sum_{\tau \in[1, t]} x_{i, \tau}=0 \quad \forall i \in I_{X}, \forall t \in[1, T] \\
& X_{i, t, \varepsilon}^{\prime}-\sum_{\tau \in[1, t]} x_{i, \tau, \varepsilon}^{\prime}=0 \quad \forall i \in I_{X^{\prime}}, \forall t \in[1, T], \forall \varepsilon \in \mathrm{E} \\
& Y_{j, t, \varepsilon}-\sum_{i \in I_{X}} n_{j, i} X_{i, t}-\sum_{i \in I_{X^{\prime}}} n_{j, i} X_{i, t, \varepsilon}^{\prime}=0 \forall j \in J, \forall t \in[1, T], \forall \varepsilon \in \mathrm{E} \\
& Y_{j, t, \varepsilon}-Y_{j, t-q_{j}, \varepsilon} \leq p_{j}+T \cdot z_{j, t, \varepsilon} \forall j \in J, \forall t \in\left[q_{j}, T\right], \forall \varepsilon \in \mathrm{E} \\
& x_{i, t} \in\{0,1\} \quad \forall i \in I_{X}, \forall t \in[1, T] \\
& x_{i, t, \varepsilon}^{\prime} \in\{0,1\} \quad \forall i \in I_{X^{\prime}}, \forall t \in[1, T], \forall \varepsilon \in \mathrm{E} \\
& z_{j, t, \varepsilon} \in\{0,1\} \quad \forall j \in J, \forall t \in\left[q_{j}, T\right], \forall \varepsilon \in \mathrm{E} \\
& Y_{j, 0, \varepsilon}=0 \quad \forall j \in J, \forall \varepsilon \in \mathrm{E}
\end{aligned}
$$


In the basic r-CSP model, the objective function (1) represents the minimization of the number of constraint violations that limit the requirements of any option $\mathrm{j} \in \mathrm{J}$ to the maximum values $p_{j} \forall j \in J$, for each demand plan $\varepsilon \in E$, and for each interval of consecutive productive cycles of length $\mathrm{q}_{\mathrm{j}}(\forall \mathrm{j} \in \mathrm{J})$. Equalities (2) impose the insertion of a vehicle to the line (regular or special), only one in each manufacturing cycle $t \in[1, T]$, and in every demand plan $\varepsilon \in E$. Equalities (3) and (4) force all the demand plans for the regular vehicles $I_{x}$ ) and the special ones $I_{0}$ to be satisfied, respectively. Equalities (5) are used to count the number of regular vehicles $i \in I_{x}$ added to line until the manufacturing cycle $t \in[1, T]$, in any demand plan $\varepsilon \in E$. Meanwhile, equalities (6), analogous to (5), refer to the special vehicles $I_{X^{\prime}}$ considering each plan $\varepsilon \in E$. Equalities (7) count the number of times that option $\mathrm{j} \in \mathrm{J}$ is required by the vehicles added consecutively to the line until a particular manufacturing cycle $t \in[1, \mathrm{~T}]$ in any demand plan $\varepsilon \in E$. Restrictions (8) determine whether a violation of the requirements restriction of every option $j \in J$ occurs in every segment, with a length $\mathrm{q}_{\mathrm{j}}(\forall \mathrm{j} \in \mathrm{J})$ of the sequence $\pi \_\varepsilon$ $(T)(\forall \varepsilon \in E)$. Conditions (9), (10), and (11) define $x_{i, t}, x_{i, t, \varepsilon}^{\prime}$, and $\mathrm{z}_{\mathrm{j}, \mathrm{t}, \mathrm{s}}$, respectively, as binary variables. Finally, equalities (12) establish, for convenience, the variables of requirement $\mathrm{Y}_{\mathrm{j}, 0, \varepsilon}(\forall \mathrm{j} \in \mathrm{J}, \forall \varepsilon \in \mathrm{E})$ as null, in the fictitious production cycle $\mathrm{t}$ $=\stackrel{\mathrm{j}, 0,8}{0}$.

The exploitation of the r-CSP model allows for a multisequence $\vec{\pi}(\mathrm{E}, \mathrm{T})$ to be obtained, and is composed of the sequences $\pi_{\varepsilon}(\mathrm{T})=\left(\pi_{1, \varepsilon}, \ldots, \pi_{\mathrm{T}, \varepsilon}\right)$ of each plan of demand $\varepsilon \in \mathrm{E}$ :

$$
\vec{\pi}(\mathrm{E}, T)=\left\{\begin{array}{c}
\pi_{1}(T) \\
\pi_{2}(T) \\
\ddot{.}(T) \\
\pi_{\varepsilon}(T) \\
\pi_{|\mathrm{E}|}(T)
\end{array}\right\}=\left\{\begin{array}{c}
\left(\pi_{1,1}, \ldots, \pi_{t, 1}, \ldots, \pi_{T, 1}\right) \\
\left(\pi_{1,2}, \ldots, \pi_{t, 2}, \ldots . \pi_{T, 2}\right) \\
\left(\pi_{1, \varepsilon}, \ldots, \pi_{t, \varepsilon}, \ldots, \pi_{T, \varepsilon}\right) \\
\left(\pi_{1, \mid \mathrm{E}}, \ldots, \pi_{t,|\mathrm{E}|}, \ldots, \pi_{T,|\mathrm{E}|}\right)
\end{array}\right\}
$$

The relationship between the vehicle type $i \in I$ and the elements $\pi_{\mathrm{t}, \varepsilon}(\forall \mathrm{t} \in[1, \mathrm{~T}], \forall \varepsilon \in \mathrm{E})$ of the multi-sequence $\vec{\pi}(\mathrm{E}, \mathrm{T})$, is established through the values adopted by the binary variables $\left.\mathrm{x}_{\mathrm{i}, \mathrm{t}}\left(\forall \mathrm{i} \in \mathrm{I} \_\mathrm{X}, \forall \mathrm{t} \in[1, \mathrm{~T}]\right) \mathrm{y} \mathrm{x}_{\mathrm{i}, \mathrm{t}, \varepsilon}^{\prime}\left(\forall \mathrm{i} \in \mathrm{I}_{\mathrm{X}^{\prime}}\right), \forall \mathrm{t} \in[1, \mathrm{~T}], \forall \varepsilon \in \mathrm{E}\right)$, present in the r-CSP model. Therefore,

$$
\begin{gathered}
x_{i, t}=1 \Rightarrow \pi_{t, \varepsilon}=i, \forall i \in I_{X}, \forall t \in[1, T], \forall \varepsilon \in \mathrm{E} \\
x_{i, t, \varepsilon}^{\prime}=1 \Rightarrow \pi_{t, \varepsilon}=i, \forall i \in I_{X^{\prime}}, \forall t \in[1, T], \forall \varepsilon \in \mathrm{E}
\end{gathered}
$$

It is noteworthy that the regular vehicles $\left(i \in I_{x}\right)$ occupy all the same positions, by type and by cycle, in all the sequences of all the demand plans, while the positions occupied by the fleets depend on each plan $\varepsilon \in E$. In other words, all the sequences $\pi_{\varepsilon}(\mathrm{T})$ will present a common part (composed by regular vehicles) and an exclusive part (composed by special vehicles).

\section{Metrics for the robustness of a multi-sequence r-CSP}

The r-CSP formulation described in the previous section corresponds to a problem of maximum satisfaction of restrictions (MAXSAT), such that its connection with the genuine CSP of Parrello, Kabat, and Vos is clear. Further, it is clear that techniques based on automatic reasoning can be used for its resolution.

Far from being satisfied with presenting and solving a MAXSAT problem and assisted by the multi-sequence production concept $\vec{\pi}(\mathrm{E}, \mathrm{T})$, in this section, several methods of measuring the quality of any solution are proposed, based on the following:

Definition 1: The multi-sequence $\vec{\pi}(\mathrm{E}, \mathrm{T})$ is strongly robust against the triple $\left(\mathrm{I}, \mathrm{J}, \mathrm{p}_{\mathrm{j}} /\left(\mathrm{q}_{\mathrm{j}}\right) \mathrm{E}\right)$, when $\mathrm{z}_{\mathrm{j}, \mathrm{t}, \varepsilon}=0$ $\forall \mathrm{j} \in J, \forall \mathrm{t} \in\left[\mathrm{q}_{\mathrm{j}}, \mathrm{T}\right], \forall \varepsilon \in E$ is satisfied.

When $\vec{\pi}(\mathrm{E}, \mathrm{T})$ does not satisfy the restrictions $\mathrm{p}_{\mathrm{j}} / \mathrm{q}_{\mathrm{j}}$ $\left(\forall \mathrm{j} \in \mathrm{J}, \forall \mathrm{t} \in\left[\mathrm{q}_{\mathrm{j}}, \mathrm{T}\right], \forall \varepsilon \in \mathrm{E}\right)$, then its quality will be evaluated through the following non-robustness metrics:

m1. Proportion of demand plans that present excessive requirement of optional elements $(j \in J)$ in a particular manufacturing cycle $\left(t \in\left[\mathrm{q}_{\mathrm{j}}, \mathrm{T}\right]\right)$ by the multi-sequence $\vec{\pi}(\mathrm{E}, \mathrm{T})$. It is useful for detecting critical demand plans.

$$
g_{1}(\vec{\pi}(\mathrm{E}, T))=\frac{1}{|\mathrm{E}|} \sum_{\varepsilon=1}^{|\mathrm{E}|} \max _{\forall j \forall t}\left\{z_{j, t, \varepsilon}\right\}
$$

$\mathrm{m} 2$. Proportion of options of set $\mathrm{J}$ that are required in excess by the multi-sequence $\vec{\pi}(\mathrm{E}, \mathrm{T})$ in a particular manufacturing cycle $\left(\mathrm{t} \in\left[\mathrm{q}_{\mathrm{j}}, \mathrm{T}\right]\right)$ and in a particular demand plan of the set E. It is useful for detecting critical optional elements.

$$
g_{2}(\vec{\pi}(\mathrm{E}, T))=\frac{1}{|J|} \sum_{j=1}^{|J|} \max _{\forall t \forall \varepsilon}\left\{z_{j, t, \varepsilon}\right\}
$$

m3. Proportion of manufacturing cycles with excessive requirement of optional elements $(j \in J)$ by the multi-sequence $\vec{\pi}(\mathrm{E}, \mathrm{T})$ in a particular demand plan of the set E. It is useful to detect critical production cycles.

$g_{3}(\vec{\pi}(\mathrm{E}, T))=\frac{1}{T} \sum_{t=1}^{T} \max _{\forall j \forall \varepsilon}\left\{z_{j, t, \varepsilon}\right\}$ 
m4. Proportion of constraints $\mathrm{p} / \mathrm{q}_{\mathrm{j}}\left(\forall \mathrm{j} \in \mathrm{J}, \forall \mathrm{t} \in\left[\mathrm{q}_{\mathrm{i}}, \mathrm{T}\right], \forall \varepsilon \in \mathrm{E} \forall-\right.$ $\mathrm{j} \in \mathrm{J})$ that violates the multi-sequence $\vec{\pi}(\mathrm{E}, \mathrm{T})$. It also represents the average proportion of manufacturing cycles with the excess of optional elements requirements $(j \in J)$ in the set of demand plans E. It is useful to determine the global non-robustness of the sequence $\vec{\pi}(\mathrm{E}, \mathrm{T})$ against all the constraints of the problem. A lower bound of metric m.4 is as follows:

$\hat{g}_{4}(\vec{\pi}(\mathrm{E}, T))=\frac{1}{|\mathrm{E}| \cdot|J| \cdot T} \sum_{j=1}^{|J|} \sum_{t=1}^{T} \sum_{\varepsilon=1}^{|\mathrm{E}|} Z_{j, t, \varepsilon}$

The refined metric function $\left.\mathrm{g}_{4}(\pi \mathrm{E}, \mathrm{T})\right)$ that strictly counts the restrictions that act on the productive cycles $\mathrm{t} \in\left[\mathrm{q}_{\mathrm{i}}, \mathrm{T}\right](\forall \mathrm{j} \in \mathrm{J})$, is defined as follows:

$$
g_{4}(\vec{\pi}(\mathrm{E}, T))=\frac{1}{|\mathrm{E}| \sum_{j=1}^{|J|}\left(T+1-q_{j}\right)} \sum_{j=1}^{|J|} \sum_{t=1}^{T} \sum_{\varepsilon=1}^{|\mathrm{E}|} Z_{j, t, \varepsilon}
$$

m5. Proportion of manufacturing cycles with maximum excess of optional elements requirements $(j \in J)$ among the set of demand plans E. It is useful to determine the most critical demand plan.

$$
g_{5}(\vec{\pi}(\mathrm{E}, T))=\max _{\forall \varepsilon}\left\{\frac{1}{\sum_{j=1}^{|J|}\left(T+1-q_{j}\right)} \sum_{j=1}^{|J|} \sum_{t=1}^{T} z_{j, t, \varepsilon}\right\}
$$

m6. Proportion of manufacturing cycles with the maximum excess requirements among the optional elements $(j \in J)$ in the set of demand plans E. It is useful to detect the most critical optional element.

$$
g_{6}(\vec{\pi}(\mathrm{E}, T))=\max _{\forall j}\left\{\frac{1}{T \cdot|\mathrm{E}|} \sum_{t=1}^{T} \sum_{\varepsilon=1}^{|\mathrm{E}|} Z_{j, t, \varepsilon}\right\}
$$

m7 Proportion of optional elements $(j \in J)$ with excessive requirements in all the demand plans of the set $\mathrm{E}$, which corresponds to the last production cycle of the most critical segments of the multi-sequence $\vec{\pi}(\mathrm{E}, \mathrm{T})$. It is useful to detect, on average, the most critical manufacturing cycle.

$$
g_{7}(\vec{\pi}(\mathrm{E}, T))=\max _{\forall t}\left\{\frac{1}{|J| \cdot|\mathrm{E}|} \sum_{j=1}^{|J|} \sum_{\varepsilon=1}^{|\mathrm{E}|} z_{j, t, \varepsilon}\right\}
$$

From the previous non-robustness metrics, it is possible to instantly define their corresponding metrics to measure the robustness of $\left.\cdot \vec{\pi}^{\prime} \mathrm{E}, \mathrm{T}\right)$ against the triple $\left(\mathrm{I}, \mathrm{J}, \mathrm{p}_{\mathrm{j}} /\left(\mathrm{q}_{\mathrm{j}}\right), \mathrm{E}\right)$. That is,

$$
r_{m}(\vec{\pi}(\mathrm{E}, T))=1-g_{m}(\vec{\pi}(\mathrm{E}, T)) m=1, \ldots, 7
$$

It is noteworthy that thus far, the terms "excessive requirements" or "excess of requirements" have been used interchangeably to reflect the violation of a particular restriction $\mathrm{p}_{\mathrm{j}} \mathrm{q}_{\mathrm{j}}$ (see restrictions (8) of the $\mathrm{r}$-CSP model), without indicating how its amount or cost is to be determined. These possible extensions of the basic r-CSP will be exposed in the subsequent work.

\section{Elementary variants of the basic r-CSP model}

Logically, the basic r-CSP model supports several variants, either by simplification or by its elementary extension. Some of them are presented below:

a. Simplification: Obviously, if only one demand plan exists (i.e., $|\mathrm{E}|=1$ ), the basic r-CSP model becomes the original CSP model. Consequently, it can be understood that the CSP is a particular case of the r-CSP; therefore, the optimal solutions of the CSP will be useful to calculate the lower bounds of the r-CSP.

b. Weighting of the excess requirement of options: If costs or weights for excess requirements $\left(\mathrm{c}_{\mathrm{j}, \mathrm{t}, \mathrm{s}}\right)$ are considered, the objective function (1) of the basic r-CSP model must be replaced by the following:

$$
\min \hat{Z}=\sum_{j \in J} \sum_{t \in\left[a_{j}, T\right]} \sum_{\varepsilon \in \mathrm{E}} c_{j, t, \varepsilon} \cdot z_{j, t, \varepsilon}
$$

where $\hat{Z}$ symbolizes the total cost for the requirement excess of optional elements of set $\mathrm{J}$ in the set of demand plans E. Here, such total cost is evaluated as a weighted sum of violations of the restrictions $\mathrm{p}_{\mathrm{j}} \mathrm{q}_{\mathrm{j}}$ of the r-CSP.

c. Mono-objective models with robustness functions of the multi-sequence: In case of incorporating the non-robustness metrics (m.1 to m.7) as elements of an optimization problem, the objective function (1) of the basic r-CSP must be replaced by one of the following functions:

$\min Z=g_{m}(\vec{\pi}(\mathrm{E}, T)) \Leftrightarrow \max Z^{\prime}=r_{m}(\vec{\pi}(\mathrm{E}, T)) \quad m=1, \ldots, 7$

d. Bi-objective models with multi-sequence robustness functions: Obviously it is neces-sary to formulate the bi-objective optimization models of robustness, replacing the objective function (1) of the basic r-CSP with any of the following functions:

$$
\left\{\begin{array}{l}
\min g_{1}(\vec{\pi}(\mathrm{E}, T)) \wedge \min g_{2}(\vec{\pi}(\mathrm{E}, T)) \\
\min g_{1}(\vec{\pi}(\mathrm{E}, T)) \wedge \min g_{3}(\vec{\pi}(\mathrm{E}, T)) \\
\min g_{2}(\vec{\pi}(\mathrm{E}, T)) \wedge \min g_{3}(\vec{\pi}(\mathrm{E}, T))
\end{array}\right\}
$$


Alternatively,

$$
\left\{\begin{array}{l}
\max r_{1}(\vec{\pi}(\mathrm{E}, T)) \wedge \max r_{2}(\vec{\pi}(\mathrm{E}, T)) \\
\max r_{1}(\vec{\pi}(\mathrm{E}, T)) \wedge \max r_{3}(\vec{\pi}(\mathrm{E}, T)) \\
\max r_{2}(\vec{\pi}(\mathrm{E}, T)) \wedge \max r_{3}(\vec{\pi}(\mathrm{E}, T))
\end{array}\right\}
$$

e. Tri-objective model with multi-sequence robustness functions: Finally, if the purpose is to represent the optimal solutions of the r-CSP on a three-dimensional Pareto front, it is reasonable to use the tri-objective functions based on the elementary metrics $\mathrm{g}_{\mathrm{m}}$ $(\vec{\pi}(\mathrm{E}, \mathrm{T}))$ or $\mathrm{r}_{\mathrm{m}}(\vec{\pi}(\mathrm{E}, \mathrm{T}))$, for $\mathrm{m}=1,2,3$. That is,

$\min g_{1}(\vec{\pi}(\mathrm{E}, T)) \wedge \min g_{2}(\vec{\pi}(\mathrm{E}, T)) \wedge \min g_{3}(\vec{\pi}(\mathrm{E}, T))$

Alternatively,

$$
\max r_{1}(\vec{\pi}(\mathrm{E}, T)) \wedge \max r_{2}(\vec{\pi}(\mathrm{E}, T)) \wedge \max r_{3}(\vec{\pi}(\mathrm{E}, T))
$$

The treatment of other variants of the r-CSP that incorporate the production costs, both in the objective function and in the restrictions of the problem, is a topic for future work.

\section{An illustrative example}

We present an example of 100 cars inspired by a classic instance of CSP literature [Little (1993)].

There are 18 types of cars available (see Figure 1), the first 14 correspond to regular vehicles (cars type 1 to 14 ), while the last 4 represent the fleets of special vehicles (cars type 15 to 18$)$.

Figure 1 Product structure and production schedule for the plan \# 8 of the robust car sequencing problem, consisting of 18 types of products and 6 component options. Option O6 is exclusive to fleet vehicles

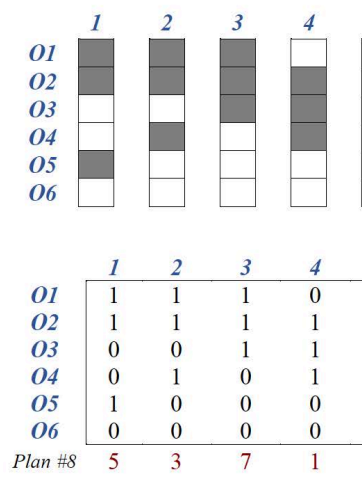

Vehicles can incorporate up to six options. The first 5 options $(\mathrm{O} 1 \ldots \mathrm{O} 5)$ come from the original instance and can be present (or not) in all the vehicles, both in the regular ones and in the fleets. On the other hand, option O6 is present in all vehicles of the fleets (value 1 in Figure 1).

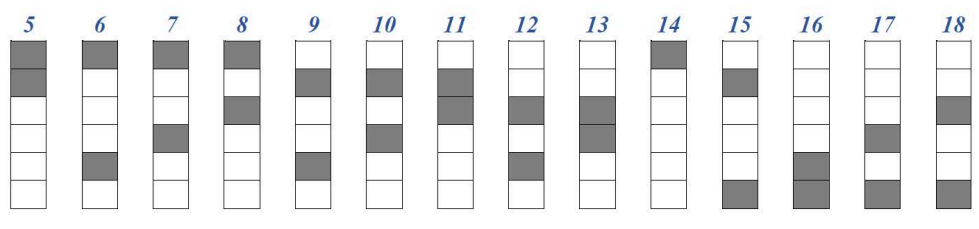

\begin{tabular}{ccccccccccccc|}
7 & 8 & 9 & 10 & 11 & 12 & 13 & 14 & 15 & 16 & 17 & 18 \\
1 & 1 & 0 & 0 & 0 & 0 & 0 & 1 & 0 & 0 & 0 & 0 \\
0 & 0 & 1 & 1 & 1 & 0 & 0 & 0 & 1 & 0 & 0 & 0 \\
0 & 1 & 0 & 0 & 1 & 1 & 1 & 0 & 0 & 0 & 0 & 1 \\
1 & 0 & 0 & 1 & 0 & 0 & 1 & 0 & 0 & 0 & 1 & 0 \\
0 & 0 & 1 & 0 & 0 & 1 & 0 & 0 & 0 & 1 & 0 & 0 \\
0 & 0 & 0 & 0 & 0 & 0 & 0 & 0 & 1 & 1 & 1 & 1 \\
11 & 5 & 4 & 6 & 12 & 1 & 1 & 5 & 9 & 5 & 12 & 1
\end{tabular}

The $\mathrm{p}_{\mathrm{j}} / \mathrm{q}_{\mathrm{j}} \quad(\mathrm{j} \in \mathrm{J})$ maximum load constraint ratios with respect to options are: $\mathrm{O} 1 \rightarrow 1 / 2, \mathrm{O} 2 \rightarrow 2 / 3, \mathrm{O} 3 \rightarrow 1 / 3, \mathrm{O} 4 \rightarrow$ $2 / 5$, O5 $\rightarrow 1 / 5$ and $\mathrm{O} 6 \rightarrow 1 / 2$; being O6 the additional option that only affects the vehicles of the fleets.

Figure 2 Solution of the instance \#8. The rows headed by the letter " $t$ " correspond to position (1 to100) and those headed by the letter "T" correspond to the vehicle types (1 to 18 ). The total demand is $\mathrm{D}=100$.
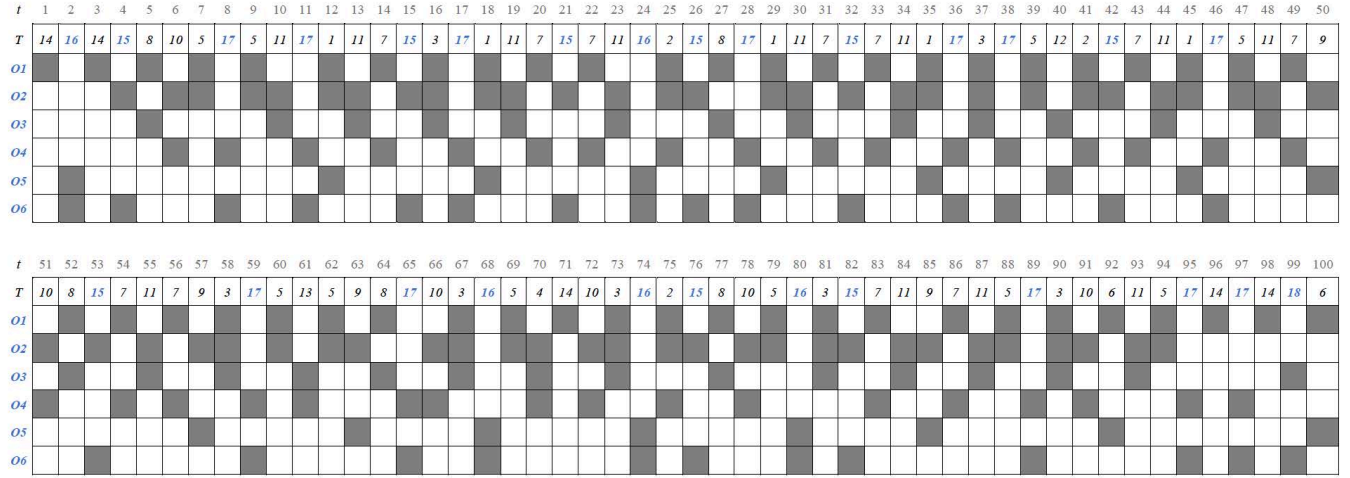

A demand plan $\varepsilon \in E$ is defined by the number of units of each type of vehicle to be manufactured, and is represent-ed by the demand plan vecto $\overrightarrow{d_{\varepsilon}}=\left(\mathrm{d}_{1, \varepsilon}, \ldots, \mathrm{d}_{\mathrm{II}, \varepsilon}\right)$. Here we will consider 22 demand plans (\# 1 to \# 22), whose values are shown in Table 1 of Annex-I. 
For example, plan \# 8 is represented by the following demand plan vector:

$$
\vec{d}_{8}=(5,3,7,1,10,2,11,5,4,6,12,1,1,5,9,5,12,1)
$$

This plan is identical to that of the original instance with a total demand $D=100$. Figure 2 shows a solution to the problem that satisfies all restrictions.
Similarly, Figures 3 and 4 show, respectively, two possible sequences for instances \# 1 and \# 22, whose demand plan vectors are:

$$
\begin{aligned}
\vec{d}_{1} & =(5,3,7,1,10,2,11,5,4,6,12,1,1,5,8,4,13,2) \\
\vec{d}_{22} & =(5,3,7,1,10,2,11,5,4,6,12,1,1,5,12,4,11,0)
\end{aligned}
$$

Figure 3 Solution of the instance $\# 1$, considering $\mathrm{p} / \mathrm{q}_{\mathrm{j}}=1 / 2$ (for $\mathrm{j}=6$ ) and $\mathrm{d}_{15,1}=8, \mathrm{~d}_{16,1}=4, \mathrm{~d}_{17,1}=13$ and $\mathrm{d}_{18,1}=2$. The total demand of vehicles in a workday is $\mathrm{D}=100\left(\mathrm{D}_{\mathrm{X}}=73, \mathrm{D}_{\mathrm{X}^{\prime}}=27\right)$.
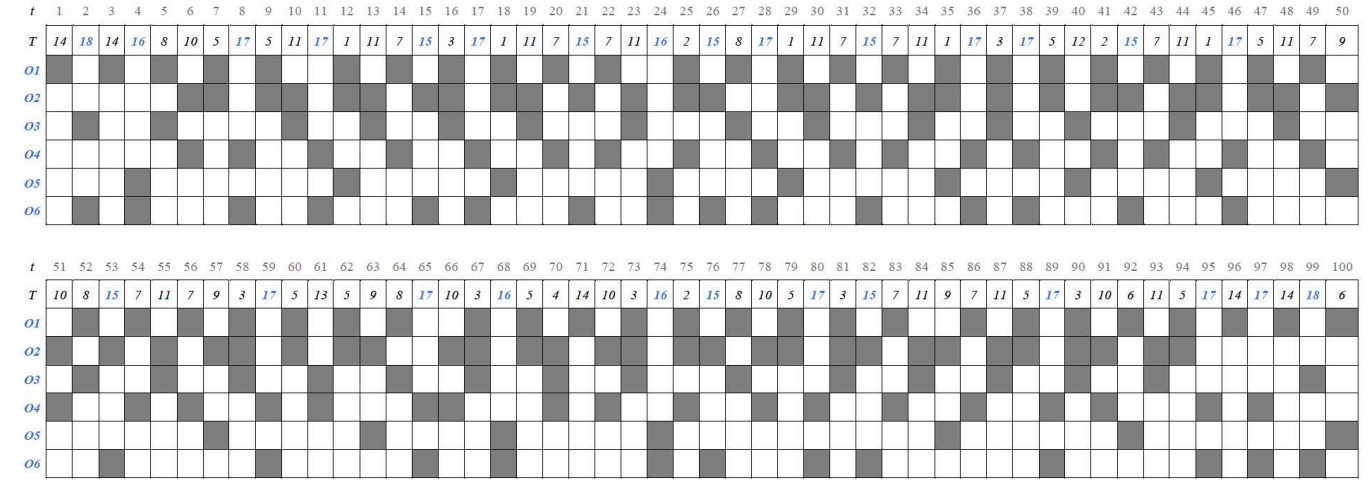

Figure 4 Solution of the instance \#22, considering $p_{j} / q_{j}=1 / 2$ (for $j=6$ ) and $\mathrm{d}_{15,22}=12, \mathrm{~d}_{16,22}=4, \mathrm{~d}_{17,22}=11$ and $\mathrm{d}_{18,22}=0$. The total demand of vehicles in a workday is $\mathrm{D}=100\left(\mathrm{D}_{\mathrm{X}}=73, \mathrm{D}_{\mathrm{X}}=27\right)$.

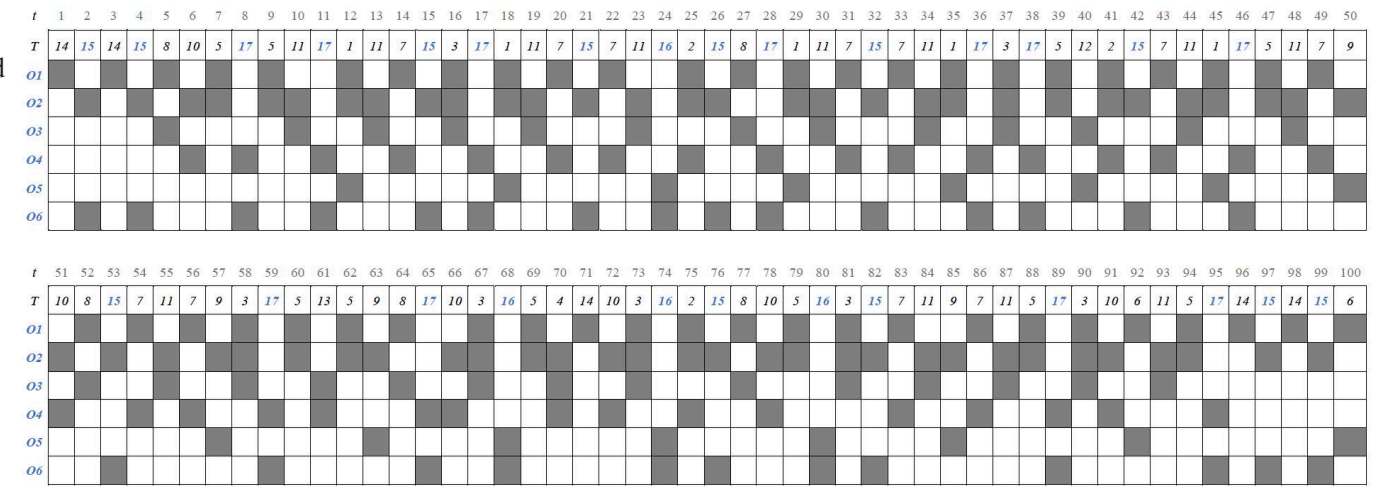

Given the conditions of the r-CSP, the demands of the regular vehicles (1 to 14) of the three previous demand plans (\# 1, \# 8 and \# 22) are identical one by one, while the demands of the vehicles of fleet (15 to 18) may vary from one plan to another.

In any case, the global demand for fleet vehicles is always the same $\left(D_{X^{\prime}}\right)=27$ cars $)$. This condition is imposed on the 22 demand plans included in Table 1 of Annex-I.
The above observation is the main characteristic of the robust car sequencing problem: the demands of the regular vehicles are fixed in all plans, while the individual demands of fleet vehicles can vary.

In summary, our example consists of finding a sequence of regular vehicles of type 1 to 14 in fixed positions (see Figure $5)$, to which is added a multi-sequence of fleet vehicles of type 15 to 18 , so that all CSP-ratios $\mathrm{p}_{\mathrm{j}} / \mathrm{q}_{\mathrm{j}}(\mathrm{j} \in \mathrm{J})$ are satisfied. 
Figure 5 Map of options for the sequences of regular vehicles of type 1 to 14 in the 22 demand plans. Free columns (positions) for fleet vehicles are headed by the letter "F".
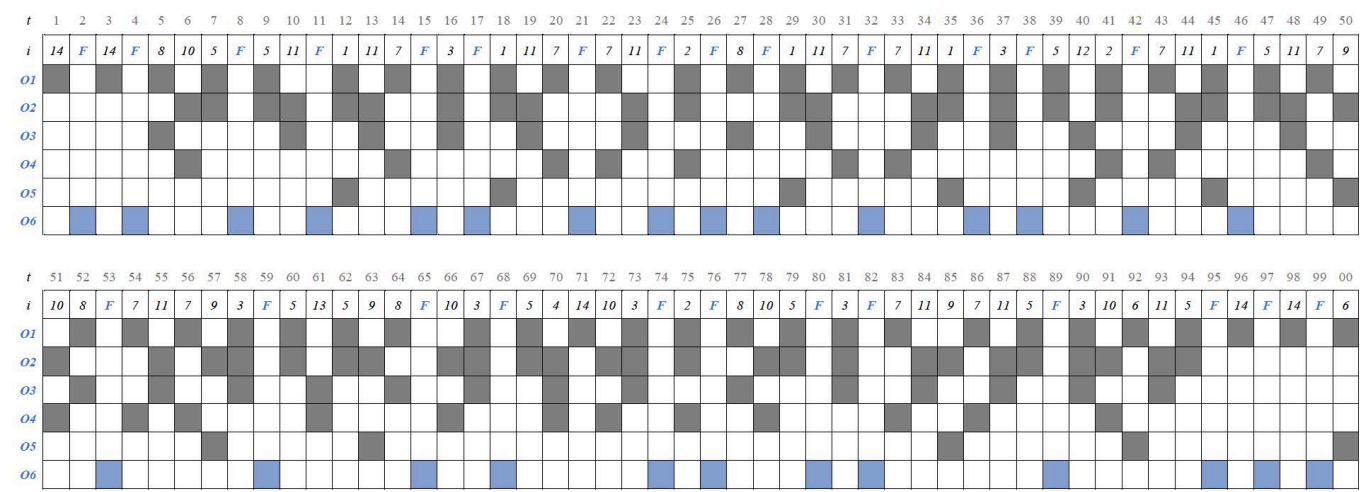

Table 2 of Annex-I shows the multi-sequence of the fleet vehicles (15 to 18 ) for the 22 production plans considered. All sequences satisfy the maximum load restrictions for options $\mathrm{O} 1$ to $\mathrm{O} 6$.

\section{Synthesis and final considerations}

This paper presents a new problem under the term, "car sequencing problem" with fleets of special vehicles and the acronym r-CSP (robust-CSP). After introducing the concept of partial demand uncertainty in special vehicle fleets with its peculiarities and present-ing the hypothesis of the problem, an optimization model based on MILP was formulat-ed, whose exploitation results in a multi-sequence of manufacturing.

The multi-sequence definition allowed for the concept of robustness to be incorporated in the sequencing problems of mixed models with partial demand uncertainty. Regard-ing the specific case of the r-CSP, 7 metrics to evaluate the non-robustness (robustness) of a solution have been proposed and could also be used as objective functions yielding several mono and multi-objective variants of the optimization problem.

The dimensions of the proposed optimization models are on the order of 23000 binary variables and 38000 explicit restrictions, considering industrial instances with 20 types of regular vehicles, 5 types of fleet vehicle, 10 types of optional elements, and 10 demand plans with 135 vehicles in a work-day. Although these dimensions are suitable to obtain solu-tions through MILP, it is convenient and advisable to also use metaheuristics to solve the r-CSP.

Furthermore, the proposals included herein could be incorporated into other sequencing problems of mixed-model production lines, or into other scheduling problems, when the appropriate circumstances arise.

Acknowledgments. This work has been funded by the Ministry of Economy and Competitiveness (Government of Spain) though the FHI-SELM2 (ref. TIN2014-57497-P) and OPTHEUS (ref. PGC2018-095080B-I00), projects, including European Regional Development Funds (ERDF).

\section{References}

Battaïa, O., Dolgui, A. (2013). "A taxonomy of line balancing problems and their solution approaches". International Journal of Production Economics 142(2), pp 259277. DOI: $10.1016 /$ j.ijpe.2012.10.020

Bautista, J. (2016). "Modelos y métricas para la versión robusta del Car Sequencing Problem con Flotas de vehículos especiales" Dirección y Organización 60 (Diciembre 2016), pp 57-65. https://www.revistadyo.es/index.php/ dyo/article/view/499

Bautista, J., Alfaro, R. (2017). "Free and regular mixed-model sequences by a linear program-assisted hybrid algorithm GRASP-LP". Progress in Artificial Intelligence 6(2), pp 159-169. DOI: 10.1007/s13748-017-0110-Z

Bautista, J., Batalla-García, C., Alfaro-Pozo, R. (2016). "Models for assembly line balancing by temporal, spatial and ergonomic risk attributes". European Journal of Operational Research 251(3), pp 814-829. DOI: 10.1016/j. ejor.2015.12.042

Bautista, J., Cano, A. (2011). "Solving mixed model sequencing problem in assembly lines with serial workstations with work overload minimization and interruption rules". European Journal of Operational Research 210(3), pp 495-513. DOI: 10.1016/j.ejor.2010.10.022

Bautista, J., Cano, A., Alfaro, A. (2012). "Modeling and solving a variant of the mixed-model sequencing problem with work overload minimisation and regularity constraints. An application in Nissan's Barcelona Plant”. Expert Systems with Applications 39 (2012), pp 1100111010. DOI: 10.1016/j.eswa.2012.03.024

Bautista, J., Companys, R., Corominas, A. (1996). "Heuristics and exact algorithms for solving the Monden problem". European Journal of Operational Research 88(1), pp 101-113. DOI: 10.1016/0377-2217(94)00165-0

Bautista, J., Companys, R., Corominas, A. (1997). "Modelling and solving the production rate variation problem (PRVP)". Top 5(2), pp 221-239. DOI: 10.1007/ BF02568551 
Bautista, J., Pereira, J., Adenso-Díaz, B. (2008.a). “A GRASP approach for the extended car sequencing problem". Journal of Scheduling 11, pp 3-16. DOI: 10.1007/ s10951-007-0046-4

Bautista, J., Pereira, J., Adenso-Díaz, B. (2008.b). “A beam search approach for the optimization version of the car sequencing problem". Annals of Operations Research 159(1), pp 233-244. DOI: 10.1007/s10479-007-0278-x

Boysen, N., Fliedner, M., Scholl, A. (2009). "Sequencing mixed-model assembly lines: Survey, classification and model critique". European Journal of Operational Research 192(2), pp 349-373. DOI: 10.1016/j. ejor.2007.09.013

Cano-Belmán, J., Ríos-Mercado, R.Z., Bautista, J. (2010). "A scatter search based hyper-heuristic for sequencing a mixed-model assembly line". Journal of Heuristics 16(6), pp 749-770. DOI: 10.1007/s10732-009-9118-2

Chica, M., Bautista, J., Cordón, O., Damas, S. (2016). “A multiobjective model and evolutionary algorithms for robust time and space assembly line balancing under uncertain demand". Omega 58, pp 55-68. DOI: 10.1016/j. omega.2015.04.003

Corominas, A., Moreno, N. (2003). "On The Relations Between Optimal Solutions For Different Types Of MinSum Balanced Jit Optimisation Problems". INFOR: Information Systems and Operational Research 41(4), pp 333-339. DOI: 10.1080/03155986.2003.11732685

Gent, I.P. (1998). "Two results on car-sequencing problems". Report University of Strathclyde, APES-02-98.

Gottlieb, J., Puchta, M., Solnon, C. (2003). "A study of greedy, local search, and ant colony optimization approaches for car sequencing problems". In Stefano Cagnoni, S. et al. (eds.): Applications of evolutionary computing, pp 246-257. Ed. Springer. DOI: 10.1007/3540-36605-9_23
Kis, T. (2004). "On the complexity of the car sequencing problem". Operations Research Letters 32 (2004), pp 331-335. DOI: 10.1016/j.orl.2003.09.003

Little, J. (1993). “A searching technique!”. OR Insight, 6(4), pp 24-31. DOI: 10.1057/ori.1993.25

Monden, Y. (1994). Toyota Production System. An Integrated Approach to Just-In-Time. Ed. Springer US. DOI: 10.1007/978-1-4615-9714-8

Morin, S., Gagné, C., Gravel, M. (2009). “Ant colony optimization with a specialized pheromone trail for the car-sequencing problem". European Journal of Operational Research 197(3), pp 1185-1191. DOI: 10.1016/j. ejor.2008.03.033

Parrello, B.D., Kabat, W.C., Wos, L. (1986). “Job-shop scheduling using automated reasoning: A case study of the car-sequencing problem". Journal of Automated reasoning 2(1), pp 1-42. DOI: 10.1007/BF00246021

Ribeiro, C.C., Aloise, D., Noronha, T.F., Rocha, C., Urrutia, S. (2008). "An efficient implementation of a VNS/ILS heuristic for a real-life car sequencing problem". European Journal of Operational Research 191(3), pp 596611. DOI: 10.1016/j.ejor.2007.02.003

Siala, M., Hebrard, E., Huguet, M.J. (2015). “A study of constraint programming heuristics for the car-sequencing problem". Engineering Applications of Artificial Intelligence 38 (2015), pp 34-44. DOI: 10.1016/j.engappai.2014.10.009

Yano, C.A., Rachamadugu, R. (1991). "Sequencing to Minimize Work Overload in Assembly Lines with Product Options" Management Science 37(5), pp 572-586. DOI: 10.1287/mnsc.37.5.572 Annex-I 
Annex-I

Table 1 Demand plans $(\varepsilon \in E$ ) for fleet vehicles of type 15 to 18 with a joint demand of 27 cars. The demand for regular vehicles is identical in all plans: $5,3,7,1,10,2,11,5,4,6,12$, $1,1,5\left(\mathrm{D}_{\mathrm{x}}=73\right)$.

\begin{tabular}{ccccccccccccccccccccccccc}
$i \backslash \varepsilon$ & $\# 1$ & $\# 2$ & $\# 3$ & $\# 4$ & $\# 5$ & $\# 6$ & $\# 7$ & $\# 8$ & $\# 9$ & $\# 10$ & $\# 11$ & $\# 12$ & $\# 13$ & $\# 14$ & $\# 15$ & $\# 16$ & $\# 17$ & $\# 18$ & $\# 19$ & $\# 20$ & $\# 21$ & $\# 22$ \\
\hline 15 & 8 & 8 & 9 & 9 & 9 & 9 & 9 & 9 & 10 & 10 & 10 & 10 & 10 & 10 & 10 & 11 & 11 & 11 & 11 & 11 & 12 & 12 \\
16 & 4 & 4 & 3 & 4 & 4 & 4 & 5 & 5 & 3 & 3 & 4 & 4 & 4 & 5 & 5 & 3 & 3 & 4 & 4 & 5 & 3 & 4 \\
17 & 13 & 14 & 14 & 12 & 13 & 14 & 11 & 12 & 13 & 14 & 11 & 12 & 13 & 11 & 12 & 12 & 13 & 11 & 12 & 11 & 12 & 11 \\
18 & 2 & 1 & 1 & 2 & 1 & 0 & 2 & 1 & 1 & 0 & 2 & 1 & 0 & 1 & 0 & 1 & 0 & 1 & 0 & 0 & 0 & 0 \\
\hline Total & 27 & 27 & 27 & 27 & 27 & 27 & 27 & 27 & 27 & 27 & 27 & 27 & 27 & 27 & 27 & 27 & 27 & 27 & 27 & 27 & 27 & 27
\end{tabular}

\begin{tabular}{llllllllllllllllllllllllllll}
$\varepsilon \backslash t$ & 2 & 4 & 8 & 11 & 15 & 17 & 21 & 24 & 26 & 28 & 32 & 36 & 38 & 42 & 46 & 53 & 59 & 65 & 68 & 74 & 76 & 80 & 82 & 89 & 95 & 97 & 99 \\
\hline
\end{tabular} $\begin{array}{lllllllllllllllllllllllllllll}1 & 18 & 16 & 17 & 17 & 15 & 17 & 15 & 16 & 15 & 17 & 15 & 17 & 17 & 15 & 17 & 15 & 17 & 17 & 16 & 16 & 15 & 17 & 15 & 17 & 17 & 17 & 18\end{array}$ $\begin{array}{lllllllllllllllllllllllllllllllll}\text { \#2 } & 17 & 16 & 17 & 17 & 15 & 17 & 15 & 16 & 15 & 17 & 15 & 17 & 17 & 15 & 17 & 15 & 17 & 17 & 16 & 16 & 15 & 17 & 15 & 17 & 17 & 17 & 18\end{array}$ $\begin{array}{lllllllllllllllllllllllllllllllll}\text { \#3 } & 17 & 15 & 17 & 17 & 15 & 17 & 15 & 16 & 15 & 17 & 15 & 17 & 17 & 15 & 17 & 15 & 17 & 17 & 16 & 16 & 15 & 17 & 15 & 17 & 17 & 17 & 18\end{array}$ $\begin{array}{lllllllllllllllllllllllllllll}\# 4 & 18 & 16 & 17 & 17 & 15 & 17 & 15 & 16 & 15 & 17 & 15 & 17 & 17 & 15 & 17 & 15 & 17 & 17 & 16 & 16 & 15 & 17 & 15 & 17 & 17 & 15 & 18\end{array}$ $\begin{array}{llllllllllllllllllllllllllllllll}\text { \#5 } & 18 & 16 & 17 & 17 & 15 & 17 & 15 & 16 & 15 & 17 & 15 & 17 & 17 & 15 & 17 & 15 & 17 & 17 & 16 & 16 & 15 & 17 & 15 & 17 & 17 & 17 & 15\end{array}$ $\begin{array}{lllllllllllllllllllllllllllll}\text { \#6 } & 17 & 16 & 17 & 17 & 15 & 17 & 15 & 16 & 15 & 17 & 15 & 17 & 17 & 15 & 17 & 15 & 17 & 17 & 16 & 16 & 15 & 17 & 15 & 17 & 17 & 17 & 15\end{array}$ $\begin{array}{lllllllllllllllllllllllllllllll}\# 7 & 18 & 16 & 17 & 17 & 15 & 17 & 15 & 16 & 15 & 17 & 15 & 17 & 17 & 15 & 17 & 15 & 17 & 17 & 16 & 16 & 15 & 16 & 15 & 17 & 17 & 15 & 18\end{array}$ $\begin{array}{llllllllllllllllllllllllllll}\text { \#8 } & 16 & 15 & 17 & 17 & 15 & 17 & 15 & 16 & 15 & 17 & 15 & 17 & 17 & 15 & 17 & 15 & 17 & 17 & 16 & 16 & 15 & 16 & 15 & 17 & 17 & 17 & 18\end{array}$

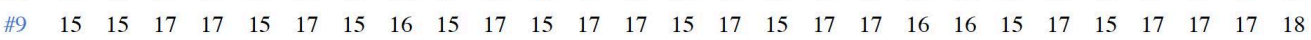
$\begin{array}{lllllllllllllllllllllllllllllllllll}\# 10 & 17 & 15 & 17 & 17 & 15 & 17 & 15 & 16 & 15 & 17 & 15 & 17 & 17 & 15 & 17 & 15 & 17 & 17 & 16 & 16 & 15 & 17 & 15 & 17 & 17 & 17 & 15\end{array}$ $\begin{array}{llllllllllllllllllllllllllllllll}\# 11 & 18 & 15 & 17 & 17 & 15 & 17 & 15 & 16 & 15 & 17 & 15 & 17 & 17 & 15 & 17 & 15 & 17 & 17 & 16 & 16 & 15 & 16 & 15 & 17 & 17 & 15 & 18\end{array}$ $\begin{array}{lllllllllllllllllllllllllllll}\# 12 & 15 & 15 & 17 & 17 & 15 & 17 & 15 & 16 & 15 & 17 & 15 & 17 & 17 & 15 & 17 & 15 & 17 & 17 & 16 & 16 & 15 & 16 & 15 & 17 & 17 & 17 & 18\end{array}$ $\begin{array}{lllllllllllllllllllllllllllll}\# 13 & 16 & 15 & 17 & 17 & 15 & 17 & 15 & 16 & 15 & 17 & 15 & 17 & 17 & 15 & 17 & 15 & 17 & 17 & 16 & 16 & 15 & 17 & 15 & 17 & 17 & 15 & 17\end{array}$ $\begin{array}{lllllllllllllllllllllllllllll}\# 14 & 16 & 15 & 17 & 17 & 15 & 17 & 15 & 16 & 15 & 17 & 15 & 17 & 17 & 15 & 17 & 15 & 17 & 17 & 16 & 16 & 15 & 16 & 15 & 17 & 17 & 15 & 18\end{array}$ $\begin{array}{lllllllllllllllllllllllllllll}\# 15 & 16 & 15 & 17 & 17 & 15 & 17 & 15 & 16 & 15 & 17 & 15 & 17 & 17 & 15 & 17 & 15 & 17 & 17 & 16 & 16 & 15 & 16 & 15 & 17 & 17 & 15 & 17\end{array}$ $\begin{array}{lllllllllllllllllllllllllllll}\# 16 & 15 & 15 & 17 & 17 & 15 & 17 & 15 & 16 & 15 & 17 & 15 & 17 & 17 & 15 & 17 & 15 & 17 & 17 & 16 & 16 & 15 & 17 & 15 & 17 & 17 & 15 & 18\end{array}$ $\begin{array}{lllllllllllllllllllllllllllll}\# 17 & 15 & 15 & 17 & 17 & 15 & 17 & 15 & 16 & 15 & 17 & 15 & 17 & 17 & 15 & 17 & 15 & 17 & 17 & 16 & 16 & 15 & 17 & 15 & 17 & 17 & 15 & 17\end{array}$ $\begin{array}{lllllllllllllllllllllllllllllll}\# 18 & 15 & 15 & 17 & 17 & 15 & 17 & 15 & 16 & 15 & 17 & 15 & 17 & 17 & 15 & 17 & 15 & 17 & 17 & 16 & 16 & 15 & 16 & 15 & 17 & 17 & 15 & 18\end{array}$ $\begin{array}{llllllllllllllllllllllllllllll}\# 19 & 16 & 15 & 17 & 17 & 15 & 17 & 15 & 16 & 15 & 17 & 15 & 17 & 17 & 15 & 17 & 15 & 17 & 17 & 16 & 16 & 15 & 17 & 15 & 17 & 17 & 15 & 15\end{array}$ $\begin{array}{llllllllllllllllllllllllllllllll}\text { \#20 } & 16 & 15 & 17 & 17 & 15 & 17 & 15 & 16 & 15 & 17 & 15 & 17 & 17 & 15 & 17 & 15 & 17 & 17 & 16 & 16 & 15 & 16 & 15 & 17 & 17 & 15 & 15\end{array}$ $\begin{array}{llllllllllllllllllllllllllll}\text { \#21 } & 15 & 15 & 17 & 17 & 15 & 17 & 15 & 16 & 15 & 17 & 15 & 17 & 17 & 15 & 17 & 15 & 17 & 17 & 16 & 16 & 15 & 17 & 15 & 17 & 17 & 15 & 15\end{array}$ \begin{tabular}{llllllllllllllllllllllllllll}
$\# 22$ & 15 & 15 & 17 & 17 & 15 & 17 & 15 & 16 & 15 & 17 & 15 & 17 & 17 & 15 & 17 & 15 & 17 & 17 & 16 & 16 & 15 & 16 & 15 & 17 & 17 & 15 & 15 \\
\hline
\end{tabular} 